\title{
Frontal alpha oscillations distinguish leaders from followers: Multivariate decoding of mutually interacting brains
}

Konvalinka, Ivana; Bauer, Markus; Stahlhut, Carsten; Hansen, Lars Kai; Roepstorff, Andreas; Frith, Chris D.

Published in:

Neurolmage

Link to article, DOI:

10.1016/j.neuroimage.2014.03.003

Publication date:

2014

Link back to DTU Orbit

Citation (APA):

Konvalinka, I., Bauer, M., Stahlhut, C., Hansen, L. K., Roepstorff, A., \& Frith, C. D. (2014). Frontal alpha oscillations distinguish leaders from followers: Multivariate decoding of mutually interacting brains. Neurolmage, 94, 79-88. https://doi.org/10.1016/j.neuroimage.2014.03.003

\section{General rights}

Copyright and moral rights for the publications made accessible in the public portal are retained by the authors and/or other copyright owners and it is a condition of accessing publications that users recognise and abide by the legal requirements associated with these rights.

- Users may download and print one copy of any publication from the public portal for the purpose of private study or research.

- You may not further distribute the material or use it for any profit-making activity or commercial gain

- You may freely distribute the URL identifying the publication in the public portal 


\title{
Frontal alpha oscillations distinguish leaders from followers:
}

\section{Multivariate decoding of mutually interacting brains}

\author{
Ivana Konvalinka ${ }^{1,2}$, Markus Bauer ${ }^{3}$, Carsten Stahlhut ${ }^{2}$, Lars Kai Hansen ${ }^{2}$, Andreas \\ Roepstorff $^{1}$, Chris D. Frith ${ }^{1,3}$ \\ ${ }^{1}$ Center of Functionally Integrative Neuroscience, Aarhus University, 8000 Aarhus, Denmark, \\ ${ }^{2}$ Section for Cognitive Systems, Department of Applied Mathematics and Computer Science, \\ Technical University of Denmark, 2800 Kongens Lyngby, Denmark, ${ }^{3}$ Wellcome Department of \\ Imaging Neuroscience, Institute of Neurology, University College London, WC1N 3BG, London, \\ UK
}

Corresponding Author: Ivana Konvalinka Current Address:

Section for Cognitive Systems, DTU Compute

Matematiktorvet, DTU Building 303 B

2800 Kongens Lyngby, Denmark

e-mail: ivana.konvalinka@gmail.com 


\begin{abstract}
:
Successful social interactions rely upon the abilities of two or more people to mutually exchange information in real-time, while simultaneously adapting to one another. The neural basis of social cognition has mostly been investigated in isolated individuals, and more recently using two-person paradigms to quantify the neuronal dynamics underlying social interaction. While several studies have shown the relevance of understanding complementary and mutually adaptive processes, the neural mechanisms underlying such coordinative behavioural patterns during joint action remain largely unknown. Here, we employed a synchronized finger-tapping task while measuring dual-EEG from pairs of human participants who either mutually adjusted to each other in an interactive task or followed a computer metronome. Neurophysiologically, the interactive condition was characterized by a stronger suppression of alpha and low-beta oscillations over motor and frontal areas in contrast to the non-interactive computer condition. A multivariate analysis of two-brain activity to classify interactive versus non-interactive trials revealed asymmetric patterns of the frontal alpha-suppression in each pair, during both task anticipation and execution, such that only one member showed the frontal component. Analysis of the behavioural data showed that this distinction coincided with the leaderfollower relationship in $8 / 9$ pairs, with the leaders characterized by the stronger frontal alpha-suppression. This suggests that leaders invest more resources in prospective planning and control. Hence our results show that the spontaneous emergence of leaderfollower relationships in dyadic interactions can be predicted from EEG recordings of brain activity prior to and during interaction. Furthermore, this emphasizes the importance of investigating complementarity in joint action.
\end{abstract}




\section{Keywords:}

social interaction; dual EEG; interpersonal coordination; multivariate decoding; leaderfollower dynamics

\section{Highlights:}

- Sensorimotor and frontal alpha oscillations suppress during dyadic interaction

- Spontaneous emergence of leader-follower relations during an interactive task

- Multivariate decoding of two brains reveals complementary neural mechanisms

- Leaders and followers can be distinguished based on frontal alpha activity 


\section{Introduction:}

When two people engage in social interaction, they exchange information with one another by producing actions and simultaneously adapting to the other person's actions via a tightly coupled alignment of perception and action within- and between-individuals (Hari and Kujala, 2009). It has been shown that both symmetrical and complementary motor adaptation of interacting partners is used when working toward a common goal (Kokal et al., 2009, Masumoto and Inui, 2013, Sacheli et al., 2013). However, the neural mechanisms underlying interpersonal real-time coordination remain largely unknown, as the methodological frameworks to study them have been underdeveloped (Konvalinka and Roepstorff, 2012, Hari et al., 2013).

Research in social cognition has only recently started to depart from studying individual minds in isolation responding to "social" stimuli, toward studies of interacting minds and brains (Sebanz et al., 2006). This movement was precipitated by the criticism that social cognition is fundamentally different when people engage in interaction, rather than remain mere observers (De Jaegher, 2009, Schilbach et al., 2013).

In particular, a number of recent studies have begun to investigate the interdependencies of neural processes in the brains of two people simultaneously as they interact (see Dumas et al., 2011, Babiloni and Astolfi, 2012, Konvalinka and Roepstorff, 2012 for reviews). These studies have provided insight into both individual neural processes during ongoing interaction, as well as interpersonal processes of two interacting brains, using hyperscanning techniques. One group of such studies has employed pseudo-interactive scenarios, scanning one person at a time in unidirectional interactions (Schippers et al., 2010, Stephens et al., 2010, Anders et al., 2011, Kuhlen et 
al., 2012), while others have measured two-brain processes during either turn-based or continuous, mutual interactions, employing fMRI (e.g. Montague et al., 2002, KingCasas et al., 2005, Saito et al., 2010), EEG (e.g. Lindenberger et al., 2009, Astolfi et al., 2010, De Vico Fallani et al., 2010, Dumas et al., 2010, Dodel et al., 2011, Yun et al., 2012), or fNIRS (e.g. Cui et al., 2012, Holper et al., 2012, Jiang et al., 2012) recordings.

More specifically, previous dual-EEG studies have consistently identified amplitude-modulation of oscillations around $10 \mathrm{~Hz}$ (alpha-band) over centro-parietal electrodes during joint attention and social coordination (Tognoli et al., 2007, Dumas et al., 2012, Lachat et al., 2012), which has also been reported in non-interactive experiments, during execution and observation of motor tasks (Cochin et al., 1999, Caetano et al., 2007) - corresponding to modulation in the rolandic mu rhythm (Gastaut, 1952). Interpersonally, two-brain studies have primarily focused on quantifying functional similarities or temporal synchronization between brains (Hasson et al., 2012) during interaction, showing evidence of inter-brain coupling when people engage in behaviourally coupled interactions (Dumas et al., 2010).

In addition to quantifying synchronized and symmetric brain-networks between brains, some dual-EEG studies have also shown asymmetric brain-coupling patterns between leader-follower participants of a dyad (Babiloni et al., 2007, Astolfi et al., 2010, Dumas et al., 2012, Sanger et al., 2012, 2013). This asymmetry has been reported either as functional connectivity (i.e. partial directed coherence) between different brain areas: prefrontal areas of a leader and ACC/parietal areas of the leader's partner in a card game (Astolfi et al., 2010); or as directed phase coupling in the alpha frequency band from frontal electrodes of leaders' brains to those of the followers' (Sanger et al., 2013). 
However, to what extent these phase-connectivity patterns might constitute a brain mechanism of social interaction (and in particular the establishment of leader and follower roles), and to what extent they may be linked to the difference in movement initiation times, remains unresolved. In contrast, we were interested whether two-brain analyses on oscillatory power - reflecting neuronal activation states - could reveal complementary patterns of individual, rather than coupled, brain mechanisms in a dyad, where the participants may take on symmetric or complementary roles.

We thus set out to investigate both the brain processes underlying mutual adaptation, and the potential inter-individual differences of interacting members within each pair. We employed a minimal interaction paradigm in order to investigate a simple interpersonal action-perception loop, whereby one person's action output became another's perceptual input, and vice-versa. This was done by asking pairs of participants to engage in a mutually interactive finger-tapping task with each other, or noninteractively with a computer metronome, whilst dual-EEG was recorded. While this synchronization paradigm typically engages symmetrical mechanisms between people when mutually adapting to each other during extended tapping (Konvalinka et al., 2009, 2010), it also allows the two members to spontaneously take on leader or follower roles, thereby potentially engaging complementary leader/follower behavioural and neural mechanisms.

A recent fMRI study investigated neural mechanisms underlying leadership, as participants engaged in a tapping paradigm with an adaptive stimulus (Fairhurst et al., 2013). The study revealed that leading and perceiving leadership correlated with rightfrontal brain activity, areas engaged in self-initiated action. Here, we wanted to develop a 
two-brain analysis, which could pick out features that could be specific to leading or following behaviour in an interactive dyad. To investigate within-pair inter-individual differences, we used a novel multivariate decoding approach, which allowed the classifier to pick up differences in brain activity during interactive versus non-interactive behaviours in either member of each pair.

The goal of our study was two-fold: to explore how ongoing brain-activity is modulated within-participants, when the task is done interactively with another person versus non-interactively with a computer; and second, to investigate how complementary forms of interactive behaviours are reflected in the brain activity of each member of a pair. 


\section{Materials and Methods:}

\subsection{Participants:}

Eighteen right-handed participants (15 male; 3 female), comprising nine pairs, volunteered for the study, recruited from Aarhus University, Denmark. They all gave written, informed consent. Ethics approval was obtained from the Science Ethics Committee for Aarhus County (Videnskabsetisk Komite for Aarhus Amt).

\subsection{Task and procedure:}

The participants were seated with their backs to one another, and received no visual feedback from each other. The experiment explored 2 conditions, 1) an interactive and 2) a computer control condition. In the interactive condition, each participant received auditory feedback only of the beats generated by the other member of the pair. In the computer condition, both participants received auditory feedback of steady, computergenerated beats. The participants never received auditory feedback of self-generated taps. The computer control was chosen because the participants received the same auditory stimulation and performed the same motor task, hence controlling for these factors. Each condition was repeated 60 times, with the order randomized. Participants were informed of their auditory feedback prior to each trial. The experimental design is shown in Figure 1 (a).

The trial was initiated by 5 steady beats from the computer, at a tempo of 120 beats per minute (bpm). The stimulus then ceased in the interactive condition, and the members only heard each other. In the computer condition, the stimulus continued at the 
steady tempo. The participants were given two instructions: to keep the given beat as precisely as possible, while at the same time synchronizing with their auditory feedback, by tapping with their right index finger for 10 beats following the 5 beat stimulus.

All participants tapped on response keys of Lumina response pads, connected to the computer via a serial port. The stimuli were sent using the Presentation software (Neurobehavioral Systems, Albany, NY, USA). One member of the pair was given two "right" earphones, and the other member was given two "left" earphones, which were connected to an earphone splitter. Therefore, tapping feedback of member one was sent to the left earphones, and feedback of member two to the right earphones, enabling the bidirectional interaction. The participants were asked to sit still, and avoid blinks and exploratory eye movements during tapping as much as possible.

\subsection{EEG recordings:}

Simultaneous EEG was recorded from both members of each pair, using two 32-channel caps with $\mathrm{Ag} / \mathrm{AgCl}$ impedance-optimized active electrodes (ActiCap, Brain Products, Gilching, Germany). The electrodes were placed at the positions of the international 1020 system, with a nasal reference. Two identical Brainamp MR amplifiers with separate grounds were used, which were optically coupled to the computer and recorded through the same software interface, ensuring synchronization between the two sets of electrodes. The recording bandwidth was set at $0.16-250 \mathrm{~Hz}$ and the data were sampled at $1000 \mathrm{~Hz}$.

\subsubsection{EEG data preprocessing:}

The data were processed and analyzed using Fieldtrip (Oostenveld et al., 2011), a 
MATLAB software toolbox for MEG/EEG analyses, developed at the Centre for Cognitive Neuroimaging of the Donders Institute for Brain, Cognition and Behaviour. All the trials were epoched from -1 to 7.5 seconds. The trials were baseline corrected in the time domain, subtracting the mean of each entire epoch, in order to remove arbitrary DC offsets.

\subsubsection{Artefact removal:}

We used Principal Component Analysis (PCA) to extract major sources of artefacts. Since in PCA the components successively extract maximal variance, this method is particularly well suited to identify sources that cause massive distortions to the recorded brain activity (Jung et al., 2000, Wallstrom et al., 2004). Moreover, PCA has been shown to effectively reduce artefacts with minimal spectral distortion (Wallstrom et al., 2004). PCA was thus computed on the data, and the topographies of the leading 8 components were visualized. One or two leading components that showed a clearly non-cortical origin (usually eye-blinks or movement artefacts in temporal electrodes) were removed.

\subsubsection{Spectral analysis:}

To quantify ongoing brain activity within participants during coordination with another person versus a non-responsive computer, a time-frequency analysis was performed on the waveforms before and during task execution (from 1 second before task onset to 7.5 seconds after). To this end, complex exponentials of frequencies from 0.5 to $30 \mathrm{~Hz}$, in steps of $0.5 \mathrm{~Hz}$, were multiplied with a Hanning taper with a fixed window length of 2 seconds. The power spectra were thus computed for each trial, and the trials were then 
averaged for each participant for the two conditions. The time range was considered up to 6 seconds, because participants tapped a shorter length on some of the trials. The power spectra of the two conditions were compared across two frequency ranges of interest: alpha (8-12) and beta $(13-30 \mathrm{~Hz})$ using the cluster permutation test. These frequency bands were selected based on previous research showing modulation of sensorimotor rhythms during social interaction or observation of motor actions (Caetano et al., 2007, Tognoli et al., 2007, Kilner et al., 2009, Dumas et al., 2012, Lachat et al., 2012).

Purely for visualization purposes (Figure 2 (a), (b)), the relative differences in power between the two conditions were calculated using the normalization index, calculated as (interactive - computer) / (interactive + computer).

\subsubsection{Statistical analysis - cluster permutation test:}

To test for significant differences between conditions that were corrected for multiple comparisons in space, time, and frequency, we performed a Monte Carlo cluster-based permutation test. This method is a widely used technique to address the multiple comparisons problem for electrophysiological data (Maris and Oostenveld, 2007). This algorithm essentially performs a mass-univariate statistical test (here, paired t-test) for all observations (in the time, frequency, and channel domain). The algorithm then forms clusters from those data-points that exceed the significance criterion (here, $p<0.05$ ) and have a neighbour in the time, frequency, and channel domain. Neighbours in the time and frequency domain are defined as adjacent points, and electrodes separated by less than 4 $\mathrm{cm}$ were defined as neighbours in the spatial domain. For each permutation, the summed statistic of all data points in a cluster is calculated and those clusters for which this 
empirically observed value (summed statistic between real conditions) exceeds the significance criterion (cluster-level alpha, here $\mathrm{p}<0.05$ ) are considered significant. The cluster-based permutation test was performed on all the 30 scalp channels (2 EOG electrodes were not included), 18 participants, and all the time points from -1 to 6 seconds with 1000 random permutations. Besides the whole-brain corrected statistical inference for the task period, we also performed a post-hoc t-test to assess whether there was also an anticipatory task-effect in the period before task execution.

\subsection{Behavioural analysis:}

The timing data of each participant's taps were used to compute the behavioural measures, for subsequent correlation with neural measures. Three sets of measures were computed, which addressed the degree of tapping coordination among participants: crosscorrelation coefficients, synchronization indices, and prediction errors. The inter-tap intervals (ITIs) were first extracted for each trial and participant. In order to quantify adaptation behaviour of each member, lag -1 and +1 correlation coefficients were computed from a cross-correlation between the inter-tap intervals of the two members in each pair. These measures are indicative of how much each participant adapts to their partner based on their partner's previous ITI, as specified in our behavioural study (Konvalinka et al., 2010). For example, lag +1 corresponds to the adaptability of member one in relation to member two (i.e. shifting the ITI time-series of member one back by 1 interval correlates to the ITI time-series of member two); and similarly, lag -1 corresponds to the adaptability of member two. In other words, if member two were to lead over member one, member one would anticipate member two's next ITI based on 
his/her previous ITI - if member two went faster on the last tap, member one would speed up on the next one. These measures were thus used to assess leader-follower behaviour in each pair, by computing one-sample t-tests on the difference between lag -1 and lag +1 coefficients for each pair.

Synchronization indices were computed statistically using the distribution of phase difference between two signals (Mardia and Jupp, 2000), corresponding to the taps of the two members in the interactive condition, and the taps of each member relative to the computer metronome in the computer condition. The index (SI) was calculated for each trial using the following formula (Tognoli et al., 2007):

$$
S I=\frac{1}{N}\left|\sum_{n=1}^{N} e^{\left(i\left(\theta_{1}\left(t_{n}\right)-\theta_{2}\left(t_{n}\right)\right)\right.}\right|
$$

where $\mathrm{N}$ is the number of taps in each trial, and $\theta_{1}$ and $\theta_{2}$ are the respective phases of each member in the pair (or the participant and the computer in the computer condition). The index is a unitless number from 0 to 1 , representing the absence of and perfect synchronization, respectively.

The prediction errors were calculated as the average difference between the timing of the produced tap and the auditory tap. Correlations were computed between the behavioural measures and the $10 \mathrm{~Hz}$ and $13-15 \mathrm{~Hz}$ effects, in the electrodes where the effects were significant.

\subsection{Multivariate classification analysis:}

One of the aims of this study was to investigate whether we could learn more about the 
complementary mechanisms of the two interacting partners within each pair, using simultaneous two-brain EEG recordings. We therefore explored whether analysis of data from pairs of brains treated as a single system would reveal additional differences between conditions. We pooled the data from both participants in each pair into one dataset, using the power centered at $10 \mathrm{~Hz}$ during tapping instances in all sixty trials per condition corresponding to each of the 30 electrodes of member one and member two as features. Hence, the data set consisted of 60 electrode features, containing data from two classes: interactive and computer. Multivariate classification analysis (Haynes and Rees, 2006) was performed using logistic regression. By fitting a logistic regression model (i.e. general linear model with a binomial cost and logit link function) (Stauffer, 2008), the two classes, 1) interactive and 2) computer control, were predicted based on the forward sequentially selected features (Guyon and Elisseeff, 2003). The classifier thus selected a subset of features from the data matrix that best predicted the class they belonged to, by sequentially selecting features until there was no improvement in prediction. This was done by starting with no features and computing cross-validation error for all subsets equal to the current feature subset plus one added feature, choosing the best subset out of all combinations and repeating the process once again with the current feature subset and one added feature. In order to assess how the results of the classification generalized to the rest of the data, a 10-fold cross validation was implemented, dividing the data into 10 segments to use as training sets. Cross-validation errors were thus computed for various feature combinations, until there was no further improvement. It was ensured that the training and test errors were not vastly different (Lemm et al., 2011). This was done across all pairs, producing an output of selected features across each cross-validation, and 
their classification training and test errors. The same procedure was repeated for power centered at $10 \mathrm{~Hz}$ features during time points corresponding to the anticipation of the task, spanning the 1-second interval prior to tapping.

Finally, this procedure was also repeated for the beta oscillations, centered at the frequency range that yielded significant effects between the interactive and computer conditions (13-15 Hz), during task execution tapping times.

\subsubsection{Permutation test for multivariate analysis:}

A permutation test was used to determine whether the electrodes selected by the classifier (which were frontal) corresponded to a leader-follower strategy. Each participant was assigned the label of leader (L) or follower (F), based on the behavioural results. The classification results were used with an ROI set on the first 7 electrodes, which were all frontal. The threshold for a feature being selected was set to $9 / 10$ cross-validation folds. The difference between the ROI classification results between member 1 and member 2 was calculated, and the difference in number of selected times summed for the L-F pairs. The L-F pairs were randomly permutated 1000 times in order to obtain the permutation distribution, which was used to calculate the statistic. This permutation test was repeated for the beta oscillations, across frontal, fronto-central, and centro-parietal electrodes. 


\section{Results:}

\subsection{Alpha and low-beta oscillations during interactive versus computer condition}

The cluster-based permutation $t$-test for dependent samples contrasting power spectra between the interactive and computer conditions revealed two significant negative clusters at $10 \mathrm{~Hz}(\mathrm{p}=0.008)$ and $13-15 \mathrm{~Hz}(\mathrm{p}=0.027)$. This indicates suppression of power during the interactive condition in contrast to the computer, from 4 to 6 seconds, thus during the entire tapping time of task execution (Figure 1 (c) and 1 (d)). The topography of $10 \mathrm{~Hz}$ effects revealed maxima in the left-central (i.e. contralateral to the tapping hand) and right-frontal electrodes. The topography of the ratio of power spectral density during the tapping period (time window from 3 to 6 seconds) against baseline (-1 to 0 seconds before the start of the trial) for each condition is shown for a single participant in Figure 1 (b). Normalized power differences ([interactive computer $] /[$ interactive + computer], see methods for details) between the two conditions over the left-central and frontal electrodes are shown in Figures 2 (a) and (b). Figure 2 (c) shows that the sensorimotor alpha power suppression began at around 2 seconds, saturated at 3 seconds when the participants began to tap, and was maintained during the whole tapping sequence for both conditions, but stronger for the interactive one. These effects resemble the sensorimotor mu-suppression at $10 \mathrm{~Hz}$ (Pfurtscheller and Lopes da Silva, 1999), but with additional frontal components. Similarly, Figures 2 (b) and (d) show that $10 \mathrm{~Hz}$ power is also suppressed over the frontal electrodes (FP1, FP2, Fz, \& F4) when the task is done interactively. This suppression is qualitatively present across the entire trial, although the difference only becomes significant from 4 seconds, as revealed by the permutation test. Additional post-hoc tests on the anticipatory period 
revealed significant $10 \mathrm{~Hz}$ suppression in right-frontal electrodes from 1-3 seconds $(\mathrm{t}=$ $1.80, \mathrm{p}<0.05)$.
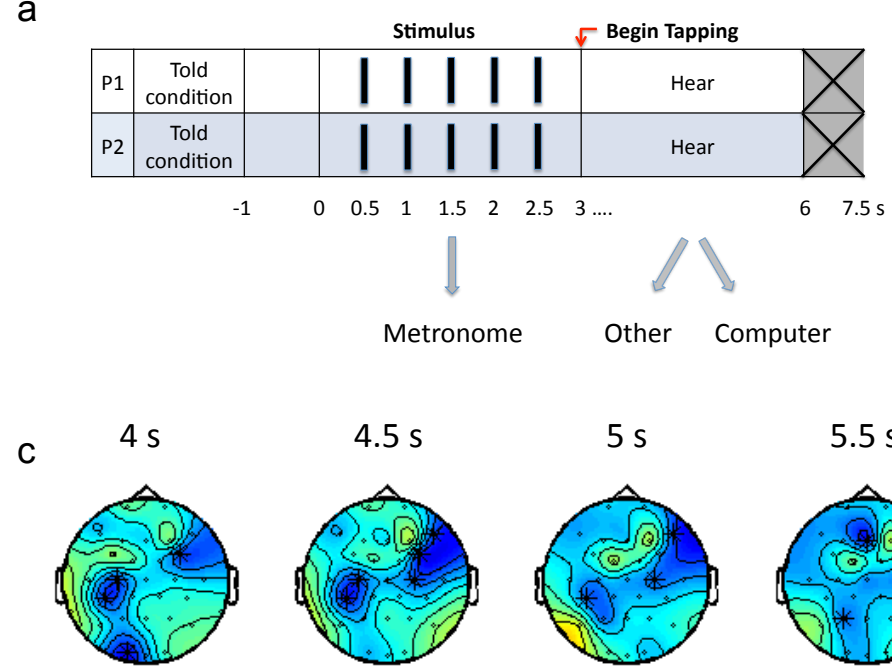

d

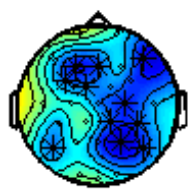

$4.5 \mathrm{~s}$
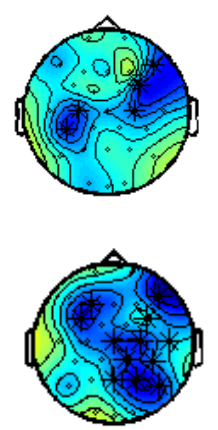
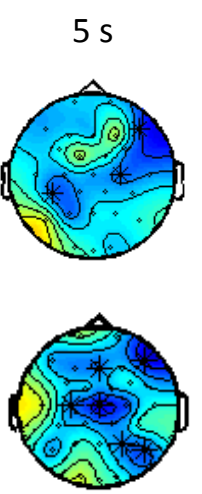

b

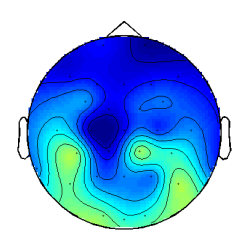

Interactive

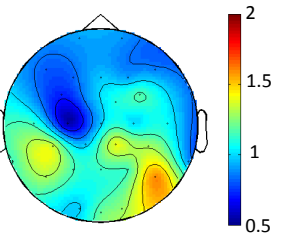

Computer
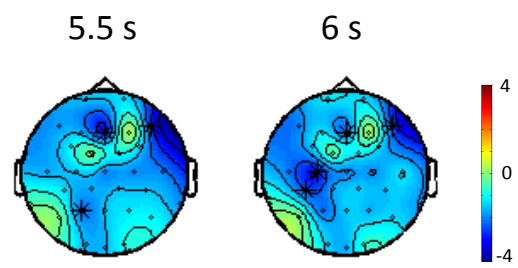

$10 \mathrm{~Hz}$
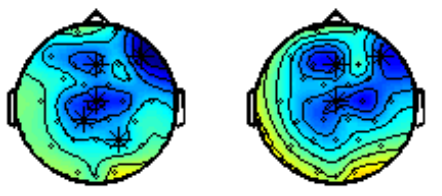

$13-15 \mathrm{~Hz}$

Figure 1 Experimental paradigm and intrapersonal results. (a) Participants are told which condition they are in (hearing other or computer) prior to task onset. The trial is initiated by 5 metronome beats at $120 \mathrm{bpm}$. The participants begin to tap at 3 seconds, while receiving auditory feedback from the other person or the computer. (b) Topography of power during tapping in relation to the baseline, as a relative difference (ratio of tapping/baseline), for a single participant. (c) Significant electrodes - those designated by '*' exceeded the $\mathrm{p}<0.05$ threshold - for the $10 \mathrm{~Hz}$ suppression when contrasting the interactive with the computer condition. (d) Significant electrodes for the $13-15 \mathrm{~Hz}$ suppression. The shown effects at 10 and $13-15 \mathrm{~Hz}$ are significant for all the time slots from 4 to 6 seconds, during task execution. The plots are a grand mean across all 18 participants. 
The normalized power-difference plot in Figure 2 (b) also reveals low beta (13-15 $\mathrm{Hz}$ ) suppression over the frontal electrodes during tapping, when doing the task with another person compared to a computer. In addition, significant clusters at $13-15 \mathrm{~Hz}$ were identified by the permutation test over the fronto-central and centro-parietal areas (Figure $1(\mathrm{c}))$.

a

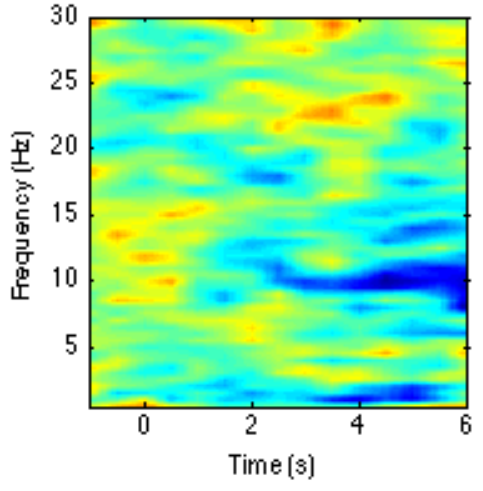

b
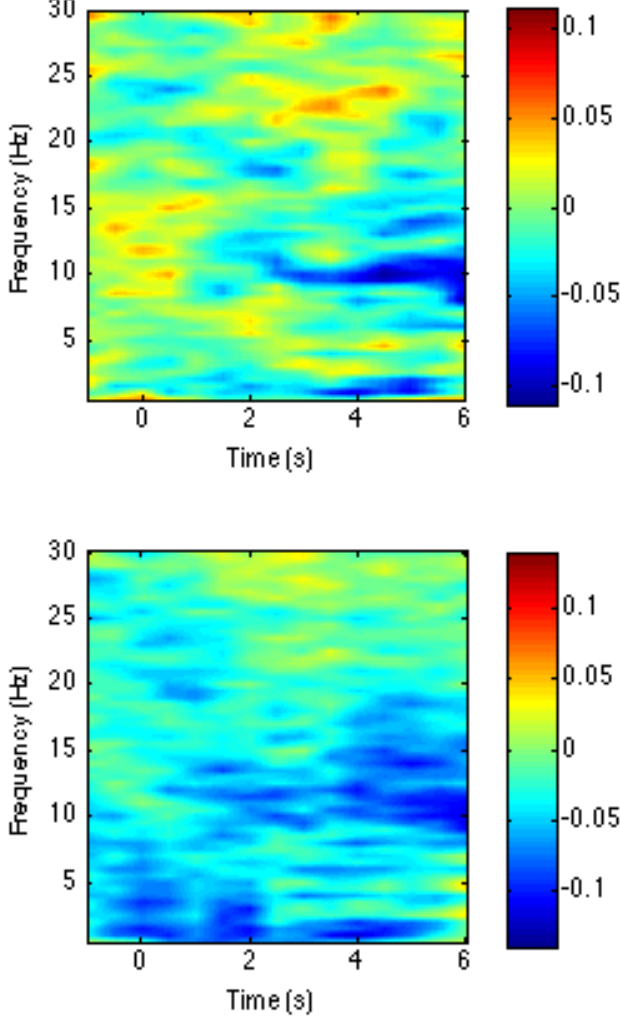

C
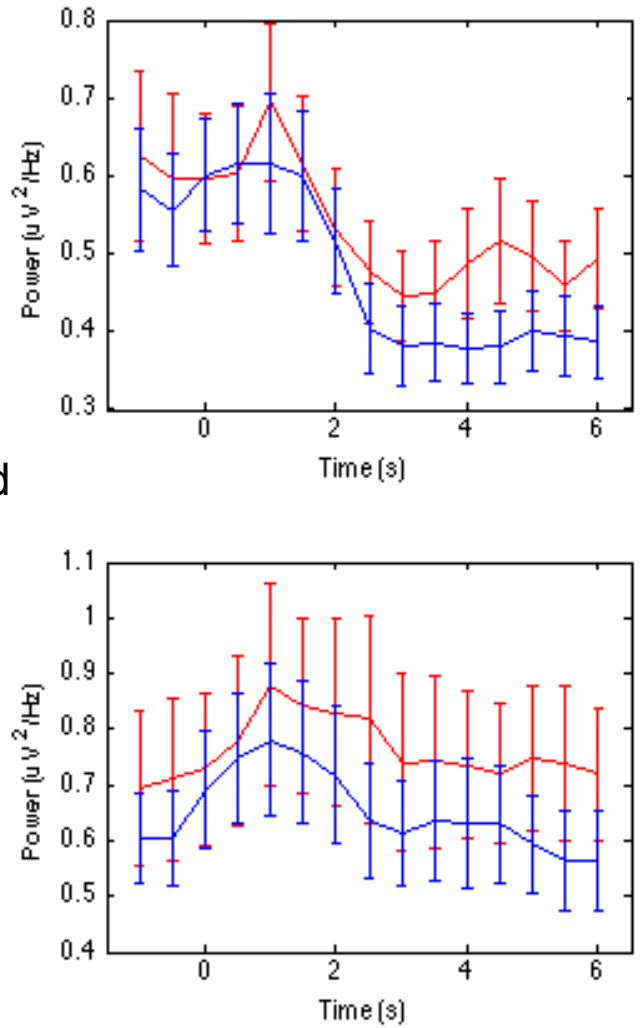

Figure $210 \mathrm{~Hz}$ modulation in interactive and computer conditions. Normalized power difference between interactive and computer conditions as a grand mean across subjects over (a) left-central electrodes, C3 and C5, and (b) frontal electrodes FP1, FP2, Fz, and F4. Time course of the mean $10 \mathrm{~Hz}$ power across (c) left-central electrodes, and (d) frontal electrodes, for the interactive (blue line) and computer (red line) conditions. The plots show the mean across participants, and standard errors of the mean at each time point. Note: tapping begins at 3 seconds. 


\subsection{Behavioural results}

In order to look for correlations between brain oscillations and behavioural data, we compared $10 \mathrm{~Hz}$ and $13-15 \mathrm{~Hz}$ power with synchronization indices of the pairs' tapping data, lag -1 and +1 correlation coefficients (corresponding to emergent leaderfollower relationships), and prediction errors. However, no significant correlations were found with any of the behavioural measures, suggesting that the suppression of 10 and 13-15 Hz oscillations has little or no relation to the strength of coupling or predictability of the stimuli.

However, behavioural analysis of lag 1 and lag -1 correlation coefficients between the inter-tap intervals of the two interacting partners in each pair revealed that lag 1 and lag -1 correlation coefficients were asymmetrically distributed in 8/9 pairs (Figure 3 (e) and 4 (b) show data across 5 different pairs; Figure 3 (d) shows sample inter-tap intervals), as revealed by significant t-tests in 8 pairs. This indicated emergence of a leader-follower dynamic in the interactive condition, and hence a difference in behaviour between the two members of most pairs, with one person being the "leader" and the other a "follower". Only one pair showed equal leading behaviour.

\subsection{Multivariate classification of paired brain data across alpha and beta oscillations}

A multivariate classification analysis was run to decode the "interactive" from the "non-interactive" computer condition, with the attempt to identify emergent patterns from the two-brain data that extend those from individual data. The results of a representative pair are displayed in Figure 3, showing electrodes (features) that contributed successfully to the classification of the interactive and computer conditions at the power of 
oscillations at $10 \mathrm{~Hz}$. The classification results reveal a strong asymmetry in $8 / 9$ pairs, such that the frontal electrodes of only one member in the pair emerged as good classifiers across all cross-validation folds (Figures 3 (a), 3 (b), 4 (a) show results of 5 different pairs). The classification accuracy of the $8 / 9$ pairs with the asymmetric pattern was $89 \%, 87 \%, 98 \%, 70 \%, 89 \%, 81 \%, 84 \%$, and $97 \%$. For the remaining pair, the frontal electrodes of both members were selected by the classifier, with an accuracy of $76 \%$. Hence, this suggests that in all but one pair engaging in dyadic interaction, only one member shows the frontal suppression.

a

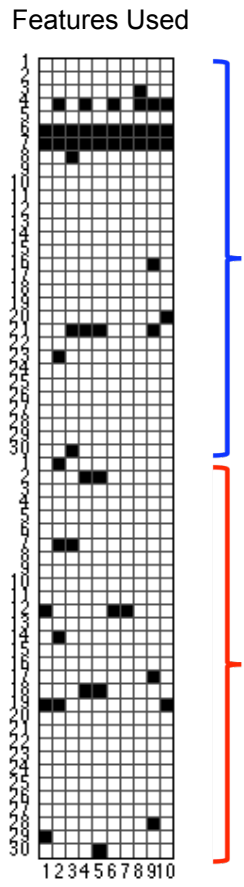

Cross-validation Fold b

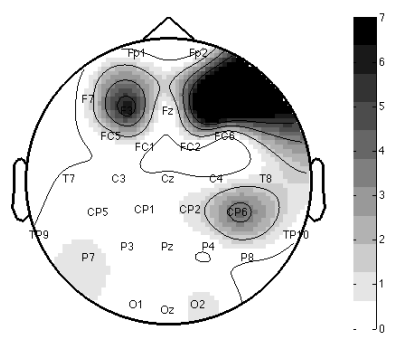

C
Member 2 Electrodes 1-30

Member 1

Electrodes 1-30

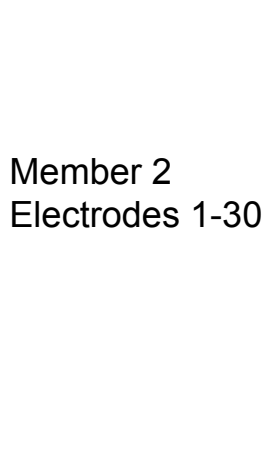

d

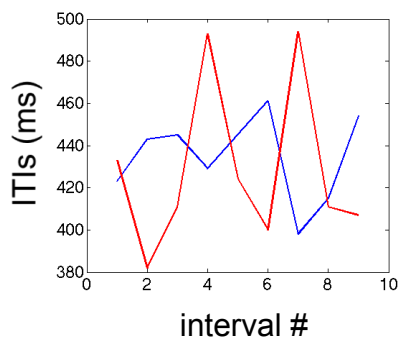

e

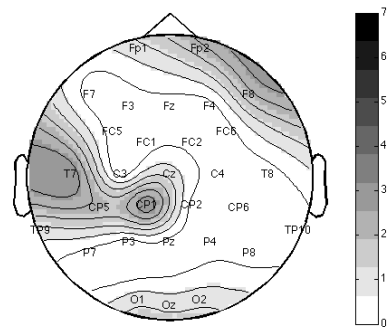

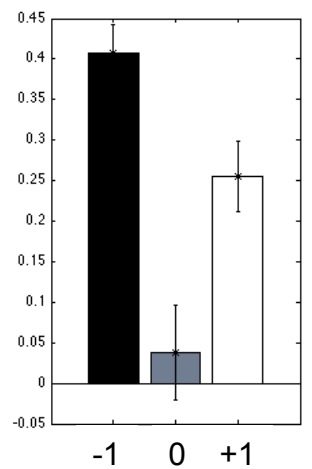

Figure 3 Classification results for $10 \mathrm{~Hz}$ oscillations of representative pair. (a) The first set of features 1-30 represent electrodes of member 1, and the second set electrodes of member 2 of the pair. The list of corresponding electrodes from 1 to 30 is: Fp1, Fp2, F7, F3, Fz, F4, F8, FC5, FC1, FC2, FC6, T7, C3, Cz, C4, T8, TP9, CP5, CP1, CP2, CP6, TP10, P7, P3, Pz, P4, P8, O1, Oz, O2. 10-fold cross validations along the x-axis, and 
features along the y-axis. Dark squares indicate that the feature was selected by the classifier during the corresponding cross-validation fold. (b) Topography of the classification results - the number of times each electrode is selected as a feature across 10 cross-validation folds - for member 1, and (c) member 2. (d) Inter-tap intervals of member 1 (blue) and member 2 (red) across a single trial. (e) Behavioural results of the pair, indicating mean lag $-1,0$, and +1 cross-correlation coefficients between inter-tap intervals of the two members. Higher lag -1 in relation to lag +1 indicates that member 2 adapts more than member 1 during the interaction.

The classification results corresponding to oscillations at low-beta frequencies (13-15 Hz) yielded a less successful classification accuracy, which was $<76 \%$ across all pairs. The data were also less consistent along the different cross-validation folds, as well as the corresponding electrodes that were selected among participants.

\subsection{Frontal alpha suppression is only associated with leaders of the interaction}

To investigate whether this tendency of one member's electrodes to drive the classifier over the other's, as seen with the classification for $10 \mathrm{~Hz}$ oscillations, is related to the interactive strategy of the participants, the results were compared to the leader/follower behaviour of the participants. As mentioned in the behavioural results, the lag -1 and lag +1 correlation coefficients between the inter-tap intervals of the two members in each pair were used as a measure of adaptability to the other person's previous inter-tap interval, hence representing leading and following behaviour. Figure 3

(e) shows correlation coefficients for the representative pair, indicating the selected member as the leader of the interaction (i.e. high correlation at lag +1 indicates member 2 as following, member 1 as leading; high correlation at lag -1 indicates member 1 as following, member 2 as leading).

In order to investigate whether this pattern was consistent among all pairs, we 
grouped all eighteen participants as either leaders or followers, depending on their lag 1 correlation coefficients. The driving member selected by the classifier corresponded to the behavioural leader of the interaction in 7/8 pairs that had a distinct dynamic (Figure 4 shows classification (a) and behavioural (b) patterns of 4 other pairs), whereas both member's electrodes were selected as features in the single pair with two behavioural leaders. Only one pair had the opposite pattern, with the frontal electrodes of the follower chosen. Figure 5 (a) shows the average classification topography (i.e. average number of times a feature is selected by the classifier) across all leaders, illustrating frontal electrodes as the selected features. The average classification topography of followers is shown in 5 (b), illustrating no notable difference between classes among the followers. For the leaders, the most common features were shown to correspond to the frontal electrodes. The corresponding normalized power difference between the two conditions for the leaders and followers is shown in Figures 5 (c) and 5 (d), respectively. The behavioural distribution of coefficients between the grouped 'leaders' and 'followers' is shown in 5 (e), with their mean adaptability captured by lag -1 and lag +1 coefficients, respectively.

The permutation test revealed statistical significance of this association, as the null hypothesis was rejected $(\mathrm{p}=0.04)$. Our results thus show distinctive neural patterns between leaders and followers during interaction.

While these patterns were distinctive at $10 \mathrm{~Hz}$, no significant differences were found between leaders and followers in the low beta frequency range. There was a slight tendency for electrodes to get more frequently selected as successful classifiers for leaders once again $(\mathrm{p}=0.19)$, but the permutation test revealed no significant association 
with leaders and any group of electrodes (frontal, fronto-central, centro-parietal).

a

Pair 1

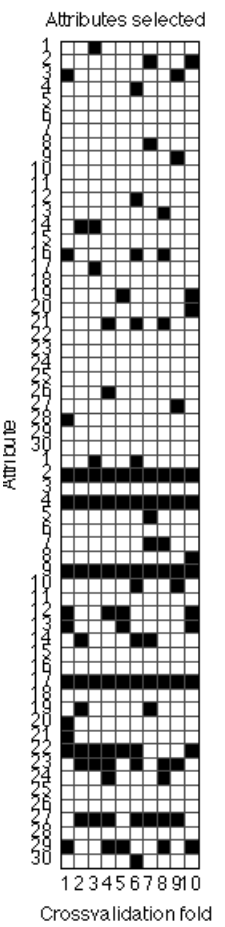

Pair 2

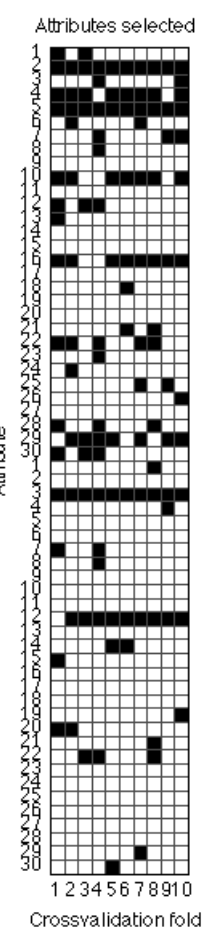

Pair 3

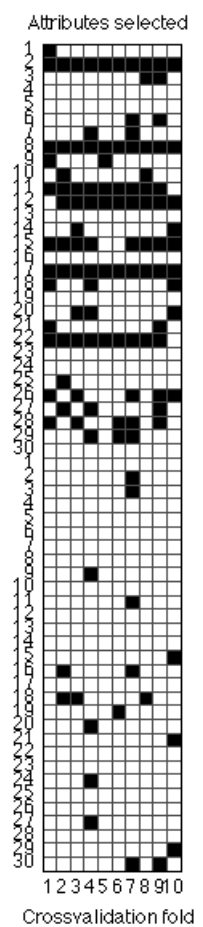

Pair 4

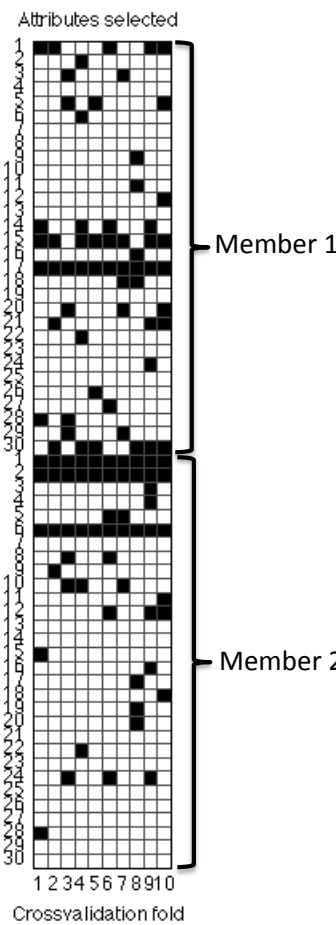

b
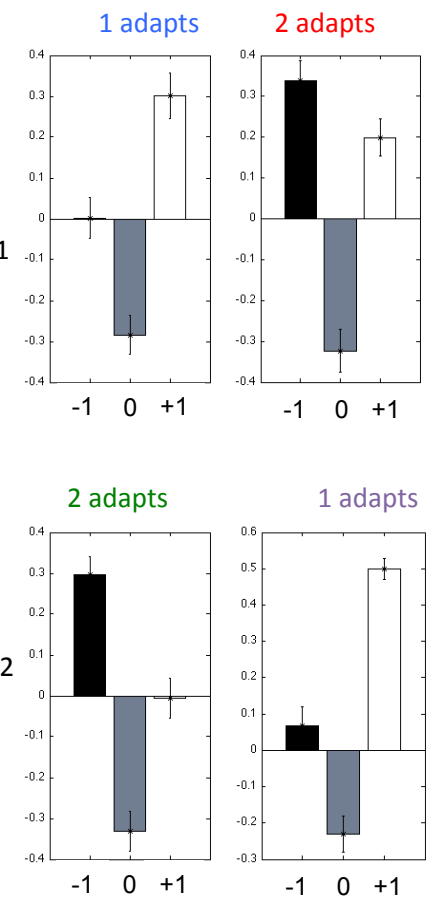

Figure 4 Classification results for $10 \mathrm{~Hz}$ oscillations of 4 other pairs. (a) The features selected by the classification analysis for member 1 (first set of features 1-30) and member 2 (second set 1-30). The electrodes correspond to those in Figure 3. (b) Behavioural results of each pair (top left - pair 1, top right - pair 2, bottom left - pair 3, bottom right - pair 4 ), indicating lag $-1,0$, and +1 cross-correlation coefficients between inter-tap intervals of the two members in each pair. 
a

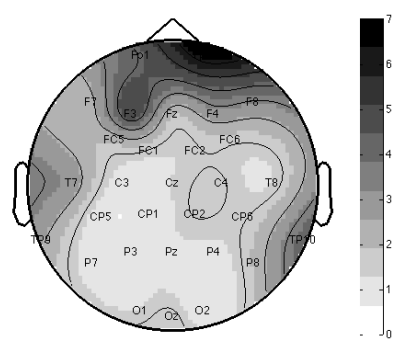

b

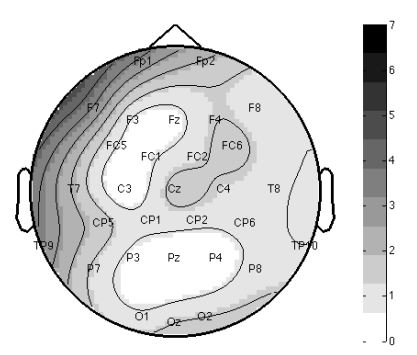

C

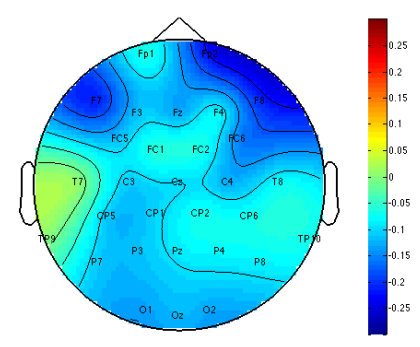

d

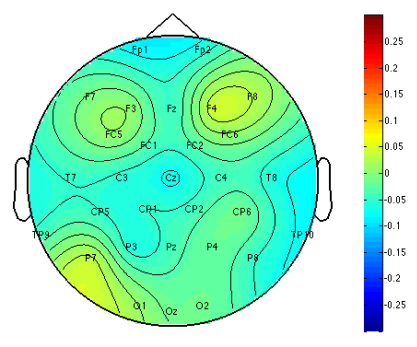

e

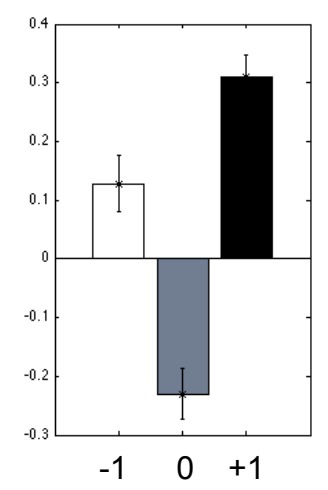

Figure $510 \mathrm{~Hz}$ results across grouped leaders and followers. (a) Topography of classification results - the mean number of times each electrode is selected as a feature across 10 cross-validation folds - across leaders of the interaction, and (b) followers. (c) and (d) display the corresponding leader/follower normalized power difference between interactive and computer conditions. (e) displays the lag $-1,0$, and +1 correlation coefficients between the inter-tap intervals of the leaders and followers. Lag -1 indicates the adaptation of the 'leaders', and lag +1 the adaptation of the 'followers'.

\subsection{Anticipatory frontal alpha oscillations}

Since participants were aware of the auditory feedback they would be receiving prior to task execution, we also investigated whether this distinction between leaders and followers at $10 \mathrm{~Hz}$ oscillations can already be made during anticipation of the task. Consistent results were obtained, with the leaders' frontal features selected in 7/9 pairs with the classification accuracy between 67 and 98\%. However, the results corresponding to the pair with two leaders, whose frontal electrodes were mutually selected during task execution, identified only one member's electrodes during anticipation of the task. 


\subsection{Addressing differences from original study (Konvalinka et al., 2010)}

It is important to note that the spontaneous emergence of a leader-follower relationship reported here is different from the findings in our original tapping study (Konvalinka et al., 2010). The behavioural results from the previous study indicated that participants adapted mutually to each other, becoming a unit of two (hyper-) followers, rather than one of them taking the lead over the other. While there were many differences between the setup of the current and the previous study, one difference that we could address was the length of tapping time. In the previous study, participants tapped 32 times in each trial, while in the current study they merely tapped 10 times. In order to see whether this leader-follower relationship is more likely to occur during the 10 taps compared to 32 taps, we ran an additional cross correlation on the first 10 taps as well as the entire trial of 32 taps of the dyads from the original study (on the data corresponding to the $120 \mathrm{bpm}$ tempo. Visually, the difference between lag +1 and lag -1 coefficients decreased across pairs after 32 taps compared to 10 , as participants showed more mutual adaptation (Figures S.1 \& S.2). While we could not address the statistical significance between adaptation of participants within each pair, given only 4 trials of the interactive (hear other) condition, we compared the absolute differences in +1 and -1 lag crosscorrelation coefficients from analysis on 10 taps versus analysis on 32 taps (Figure S.3). The difference between lag +1 and lag -1 coefficients was significantly higher after 10 taps than after 32 (paired t-test, $\mathrm{p}=0.019$ ).

Therefore, participants in the original study became more mutually adaptive over time. This explains why we might see differences in leader-follower behaviour between the two studies - given shorter trials, people tend to be more asymmetrical in their 
adaptation.

\section{Discussion:}

In this study, we presented a minimal paradigm for studying between-person actionperception coupling. The study extends previous work on mutually interacting brains, with a new analysis approach aiming to explain the complementary brain mechanisms of two-people as they engage in interaction. The multivariate decoding analysis of two-brain data enables statistical distinction to be made between neural processes of each member of the pair, showing that frontal alpha suppression is selectively found in the participant leading the interaction.

Consistent with previous studies of neural oscillations during joint attention (Lachat et al., 2012), coordination (Tognoli et al., 2007, Dumas et al., 2012, Naeem et al., 2012a, b), and observation (Cochin et al., 1999, Caetano et al., 2007), $10 \mathrm{~Hz}$ suppression was found over left-central electrodes when interacting with another person versus a nonresponsive computer, with additional frontal components. While mu-rhythm suppression is well known to occur over the bilateral sensorimotor cortex (but stronger over the contralateral cortex) when engaging in motor activity in contrast to rest (Gastaut, 1952, Gastaut and Bert, 1954, Pfurtscheller and Lopes da Silva, 1999), it has also been found during action observation (Hari et al., 1998, Cochin et al., 1999, Caetano et al., 2007), and is hence thought to be part of a common coding mechanism of perception and action (Hari, 2006, de Lange et al., 2008).

In this experiment, we examined a simple action-perception loop, where one person's motor output was another's perceptual input, and vice-versa. Hence, the scenario 
enabled participants to either follow the other person's beat, by adjusting their immediate tapping time corresponding to the speed of the other person's previous tap; to try to take the lead over the other member, e.g. by monitoring their own tapping more than the other's; or to find a balance between their own beat and the other's output. Notably, our results show that both participants of the leader-follower pairs display adaptive behaviour (Figure 3 (e), 4, 5 (e)), as seen in the behavioural study (Konvalinka et al., 2010). However, the two interactive partners do not take an entirely symmetrical position, as the 'follower' is the more adaptive one in all but one pair (Fairhurst et al., 2013), indicating spontaneous emergence of a leader-follower dynamic. This seems contradictory to the findings from our previous study. However, additional analysis showed that participants' relationship resembled that of a leader and a follower even in the original study if only the first 10 taps are analyzed. We can therefore conclude that dyads become more mutually adaptive over time, but we cannot say what factors determine whether people become leaders or followers. This aspect is left for future research.

Leader-follower dynamics have been investigated in behavioural studies before (Noy et al., 2011, Sacheli et al., 2013, Wing et al., 2014), and previous work on agency has addressed the roles of imitators (i.e. followers) and initiators (i.e. leaders) of action in reciprocal imitation paradigms in both interactive (Dumas et al., 2012, Guionnet et al., 2012) and non-interactive scenarios (Decety et al., 2002). These studies have shown activation of the inferior parietal cortex during initiation and imitation of stimuli, in the absence of interaction (Decety et al., 2002), as well as recruitment of fronto-parietal regions during interactive imitation (Guionnet et al., 2012). In contrast to these studies, the aim of the current study was to develop a two-brain analysis, which uses data from 
both brains to uncover the complementary neural mechanisms of spontaneous leaderfollower dyads in a tightly-coupled coordinative task.

In this experiment, tapping with another person engaged the behaviour of one leader and one follower, whereas tapping with a computer engaged only behaviour of a follower who adjusted to a non-responsive beat without the ability to take the lead. Our results show that differences in frontal $10 \mathrm{~Hz}$ oscillations only of leaders of the interaction distinguish the two conditions. The fact that the classifier in our experiment does not pick up on any significant features distinguishing the two conditions in followers could be explained by the presence of following behaviour in both conditions. This suggests that frontal $10 \mathrm{~Hz}$ amplitude-modulation could correspond to leading behaviour during interpersonal coordination. This is consistent with recent findings by Fairhurst and colleagues (2013), who showed that leading (perceiving leadership as well as tapping with an overly adaptive stimulus) correlated with right lateralized frontal activation, measured using fMRI.

Importantly, in addition to frontal alpha-effects during task execution, classification analysis carried out on the interval corresponding to 1 second prior to tapping shows that anticipatory $10 \mathrm{~Hz}$ suppression is also associated with the leaders of the interaction. This suggests that mentally anticipating an interactive task may set the system in an enhanced readiness state that enables the participant to quickly gain dominance over his/her partner. This is in line with previous work showing that top-down predictive mechanisms play a crucial role in facilitating successful joint coordination (Knoblich and Jordan, 2003, Pecenka and Keller, 2011, Kourtis et al., 2012, Vesper et al., 2013). 
Previous research has suggested that alpha band oscillations are closely related to functions of attention and prediction (Klimesch et al., 2007). Alpha suppression, or eventrelated desynchronization, is typically seen as the consequence of excitatory input (or release from inhibition) (Pfurtscheller and Lopes da Silva, 1999, Jensen and Mazaheri, 2010) from either bottom-up or top-down inputs (de Lange et al., 2008, Bauer et al., 2012), and therefore considered to reflect an increase of neural activity - for example, over the sensorimotor cortex during production of movements (Kuhlman, 1978). In the current study, the decrease in $10 \mathrm{~Hz}$ power over left-central regions, seen across all participants, would thus suggest more attention allocated to the action-perception coupling aspects of the interactive task.

In addition to sensorimotor effects, suppression of oscillatory alpha-power over frontal sensors has previously been reported during movement planning and execution (e.g. Verhagen et al., 2012). A follow-up study showed that stronger alpha-suppression over parietofrontal regions coincided with increasing task complexity, an effect that was seen prior to movement onset (Verhagen et al., 2013). This suggests a role of frontal alpha-suppression in action planning and execution.

Finally, functional imaging studies have shown that planning and control abilities are typically associated with the prefrontal cortex (PFC) (Miller and Cohen, 2001). Specifically, previous research has suggested that the PFC has a role in self-other processing, with the ventral-medial PFC more strongly engaged in self-processing, and the dorsal PFC engaged during processing related to others (Mitchell et al., 2006, Sui et al., 2013).

Taken together, these findings suggest that the frontal alpha-suppression found 
during anticipation and execution could reflect activation of frontal areas during interactive task planning and control. Since this frontal suppression is particularly enhanced in leaders, this could be interpreted as more resources allocated to selfprocessing, allowing the leaders to attend to their own beat rather than merely monitor the output of their partner. However, since we do not have source localization data, we cannot pinpoint the exact area of this frontal suppression. Notably, this study supports recent findings showing activation of frontal brain areas (i.e. involved in cognitive control and self-processing) in leaders, measured using fMRI (Fairhurst et al., 2013).

While it could be argued that the interactive task in this experiment is also more cognitively demanding than tapping with a predictable metronome, we did not find any correlation between $10 \mathrm{~Hz}$ suppression and prediction error. In addition, the presence of the effect during task anticipation also rules out that the $10 \mathrm{~Hz}$ suppression can be explained purely by cognitive load effects related to increased precision of motor control.

We therefore propose that the frontal $10 \mathrm{~Hz}$ suppression is related to the increased self-monitoring required in leaders, at least in this simple tapping task. In the interactive condition, both participants made predictions about the behaviour of the other, as well as about the sensory consequences of their own action. After each tap, they mutually adjusted their future prediction and adapted their next action accordingly. In the computer condition, by contrast, they only had to monitor their tapping with respect to the external beat, which was at a steady rhythm and therefore required no updating. Leading the interaction thus required prospective planning and control, rather than the mere monitoring required of a follower. These aspects of leading the interaction may be enabled by enhanced cognitive efforts such as readiness for the task and increased self- 
monitoring, as reflected by stronger frontal alpha-suppression.

We were not able to find any consistency in the two-brain analyses of beta oscillations, and can only speculate the reasons for this. It could be that the individual analyses showed more suppression of power in low beta oscillations in the interactive condition due to top-down influences - knowing (or rather, believing) that the partner they were interacting with was a human versus a computer; or that the role of beta suppression is related to aspects of the experience of interacting with another person. However, we have no evidence that beta oscillations were linked to leading or following behaviour.

An important question for future research is whether the observed differences in participants are due to state-fluctuations that emerge from the particular combination of participants, or rather are stable traits that might generalize to more complex social interactions. Moreover, our paradigm and methodological approach leave considerable opportunity for further exploration. It could be used to disentangle how much of this effect is related to the belief that one is interacting with another person, and how much is due to how the other person adapts, by experimentally manipulating the belief of the interacting partner.

In addition, our multivariate approach is particularly well suited for exploratory analysis of two-person brain signals. Given the methodological and conceptual challenges of studying brain mechanisms of social interaction, hyperscanning still has a long way to go, and explorative analyses can be useful in decoding both synchronized and complementary mechanisms in two-brain data during more natural interactions (Konvalinka and Roepstorff, 2012). While our original hypothesis was not regarding 
differences in brain signals between leaders and followers, this distinction was uncovered given our data-driven approach. Crucially, development of multivariate approaches that can tap into interpersonal brain mechanisms during social interaction is necessary for future work.

The real challenge that remains is extending a) low-level interactive tasks to faceto-face interactions involving higher-level social tasks, and b) multivariate methods to explore changes in neural dynamics during the course of a trial. We suggest that the key message to emerge from this study of joint action is the demonstration of the importance of complementarity, rather than synchrony, at both the behavioural and neural level. 


\section{Acknowledgments}

This work was supported by the Danish National Research Foundation through the Interacting Minds project, the MINDLab UNIK initiative at Aarhus University funded by the Danish Ministry of Science, Technology and Innovation, the Danish Lundbeckfonden through CIMBI Center for Integrated Molecular Brain Imaging, and the Danish Council for Independent Research - Technology and Production Sciences grant awarded to I. K. The authors thank Joshua Skewes and Dan Bang for assistance with the experimental setup and data collection, and Peter Vuust for helpful discussions.

\section{Conflict of Interest}

The authors declare no competing financial interests. 


\section{References}

Anders S, Heinzle J, Weiskopf N, Ethofer T, Haynes JD (2011) Flow of affective information between communicating brains. Neuroimage 54:439-446.

Astolfi L, Toppi J, De Vico Fallani F, Vecchiato G, Salinari S, Mattia D, Cincotti F, Babiloni F (2010) Neuroelectrical hyperscanning measures simultaneous brain activity in humans. Brain Topogr 23:243-256.

Babiloni F, Astolfi L (2012) Social neuroscience and hyperscanning techniques: Past, present and future. Neurosci Biobehav Rev.

Babiloni F, Cincotti F, Mattia D, De Vico Fallani F, Tocci A, Bianchi L, Salinari S, Marciani M, Colosimo A, Astolfi L (2007) High resolution EEG hyperscanning during a card game. Conf Proc IEEE Eng Med Biol Soc 2007:4957-4960.

Bauer M, Kennett S, Driver J (2012) Attentional selection of location and modality in vision and touch modulates low-frequency activity in associated sensory cortices. J Neurophysiol 107:2342-2351.

Caetano G, Jousmaki V, Hari R (2007) Actor's and observer's primary motor cortices stabilize similarly after seen or heard motor actions. Proc Natl Acad Sci U S A 104:9058-9062.

Cochin S, Barthelemy C, Roux S, Martineau J (1999) Observation and execution of movement: similarities demonstrated by quantified electroencephalography. Eur J Neurosci 11:1839-1842.

Cui X, Bryant DM, Reiss AL (2012) NIRS-based hyperscanning reveals increased interpersonal coherence in superior frontal cortex during cooperation. Neuroimage 59:2430-2437.

De Jaegher H (2009) Social understanding through direct perception? Yes, by interacting. Conscious Cogn 18:535-542; discussion 543-550.

de Lange FP, Jensen O, Bauer M, Toni I (2008) Interactions between posterior gamma and frontal alpha/beta oscillations during imagined actions. Front Hum Neurosci 2:7.

De Vico Fallani F, Nicosia V, Sinatra R, Astolfi L, Cincotti F, Mattia D, Wilke C, Doud A, Latora V, He B, Babiloni F (2010) Defecting or not defecting: how to "read" human behavior during cooperative games by EEG measurements. PLoS One 5:e14187.

Decety J, Chaminade T, Grezes J, Meltzoff AN (2002) A PET exploration of the neural mechanisms involved in reciprocal imitation. Neuroimage 15:265-272.

Dodel S, Cohn J, Mersmann J, Luu P, Forsythe C, Jirsa V (2011) Brain signatures of team performance. In: Lecture notes in computer science, vol. 6780, pp 288-297.

Dumas G, Lachat F, Martinerie J, Nadel J, George N (2011) From social behaviour to brain synchronization: Review and perspectives in hyperscanning. IRBM 32:4853.

Dumas G, Martinerie J, Soussignan R, Nadel J (2012) Does the brain know who is at the origin of what in an imitative interaction? Front Hum Neurosci 6:128.

Dumas G, Nadel J, Soussignan R, Martinerie J, Garnero L (2010) Inter-brain synchronization during social interaction. PLoS One 5:e12166. 
Fairhurst MT, Janata P, Keller PE (2013) Leading the follower: An fMRI investigation of dynamic cooperativity and leader-follower strategies in synchronization with an adaptive virtual partner. Neuroimage 84C:688-697.

Gastaut H (1952) Etude electrocorticographique de la reactivite des rythmes rolandiques. Rev Neurologiq 87:176-182.

Gastaut H, Bert J (1954) EEG changes during cinematographic presentation. Electroencephalogr Clin Neurophysiol 6:433-444.

Guionnet S, Nadel J, Bertasi E, Sperduti M, Delaveau P, Fossati P (2012) Reciprocal imitation: toward a neural basis of social interaction. Cereb Cortex 22:971-978.

Guyon I, Elisseeff A (2003) An introduction to variable and feature selection. Journal of Machine Learning Research 3:1157-1182.

Hari R (2006) Action-perception connection and the cortical mu rhythm. Prog Brain Res 159:253-260.

Hari R, Forss N, Avikainen S, Kirveskari E, Salenius S, Rizzolatti G (1998) Activation of human primary motor cortex during action observation: a neuromagnetic study. Proc Natl Acad Sci U S A 95:15061-15065.

Hari R, Himberg T, Nummenmaa L, Hamalainen M, Parkkonen L (2013) Synchrony of brains and bodies during implicit interpersonal interaction. Trends Cogn Sci.

Hari R, Kujala MV (2009) Brain basis of human social interaction: from concepts to brain imaging. Physiol Rev 89:453-479.

Hasson U, Ghazanfar AA, Galantucci B, Garrod S, Keysers C (2012) Brain-to-brain coupling: a mechanism for creating and sharing a social world. Trends Cogn Sci.

Haynes J, Rees G (2006) Decoding mental states from brain activity in humans. Nat Neurosci 7:523-534.

Holper L, Scholkmann F, Wolf M (2012) Between-brain connectivity during imitation measured by fNIRS. Neuroimage 63:212-222.

Jensen O, Mazaheri A (2010) Shaping functional architecture by oscillatory alpha activity: gating by inhibition. Front Hum Neurosci 4:186.

Jiang J, Dai B, Peng D, Zhu C, Liu L, Lu C (2012) Neural synchronization during faceto-face communication. J Neurosci 32:16064-16069.

Jung TP, Makeig S, Humphries C, Lee TW, McKeown MJ, Iragui V, Sejnowski TJ (2000) Removing electroencephalographic artifacts by blind source separation. Psychophysiology 37:163-178.

Kilner JM, Marchant JL, Frith CD (2009) Relationship between activity in human primary motor cortex during action observation and the mirror neuron system. PLoS One 4:e4925.

King-Casas B, Tomlin D, Anen C, Camerer CF, Quartz SR, Montague PR (2005) Getting to know you: reputation and trust in a two-person economic exchange. Science 308:78-83.

Klimesch W, Sauseng P, Hanslmayr S (2007) EEG alpha oscillations: the inhibitiontiming hypothesis. Brain Res Rev 53:63-88.

Knoblich G, Jordan JS (2003) Action coordination in groups and individuals: Learning anticipatory control. Journal of Experimental Psychology-Learning Memory and Cognition 29:1006-1016.

Kokal I, Gazzola V, Keysers C (2009) Acting together in and beyond the mirror neuron system. Neuroimage 47:2046-2056. 
Konvalinka I, Roepstorff A (2012) The two-brain approach: how can mutually interacting brains teach us something about social interaction? Front Hum Neurosci 6:215.

Konvalinka I, Vuust P, Roepstorff A, Frith CD (2009) A coupled oscillator model of interactive tapping. In: Proceedings of the European Society for the Cognitive Sciences of Music (ESCOM) Jyvaskyla, Finland.

Konvalinka I, Vuust P, Roepstorff A, Frith CD (2010) Follow you, follow me: continuous mutual prediction and adaptation in joint tapping. Q J Exp Psychol (Colchester) 63:2220-2230.

Kourtis D, Sebanz N, Knoblich G (2012) Predictive representation of other people's actions in joint action planning: An EEG study. Soc Neurosci.

Kuhlen AK, Allefeld C, Haynes JD (2012) Content-specific coordination of listeners' to speakers' EEG during communication. Front Hum Neurosci 6:266.

Kuhlman WN (1978) Functional topography of the human mu rhythm. Electroencephalogr Clin Neurophysiol 44:83-93.

Lachat F, Hugueville L, Lemarechal JD, Conty L, George N (2012) Oscillatory Brain Correlates of Live Joint Attention: A Dual-EEG Study. Front Hum Neurosci 6:156.

Lemm S, Blankertz B, Dickhaus T, Muller KR (2011) Introduction to machine learning for brain imaging. Neuroimage 56:387-399.

Lindenberger U, Li SC, Gruber W, Muller V (2009) Brains swinging in concert: cortical phase synchronization while playing guitar. BMC Neurosci 10:22.

Mardia KV, Jupp PE (2000) Directional Statistics: Wiley, Chichester, UK.

Maris E, Oostenveld R (2007) Nonparametric statistical testing of EEG- and MEG-data. J Neurosci Methods 164:177-190.

Masumoto J, Inui N (2013) Two heads are better than one: both complementary and synchronous strategies facilitate joint action. J Neurophysiol 109:1307-1314.

Miller EK, Cohen JD (2001) An integrative theory of prefrontal cortex function. Annu Rev Neurosci 24:167-202.

Mitchell JP, Macrae CN, Banaji MR (2006) Dissociable medial prefrontal contributions to judgments of similar and dissimilar others. Neuron 50:655-663.

Montague PR, Berns GS, Cohen JD, McClure SM, Pagnoni G, Dhamala M, Wiest MC, Karpov I, King RD, Apple N, Fisher RE (2002) Hyperscanning: simultaneous fMRI during linked social interactions. Neuroimage 16:1159-1164.

Naeem M, Prasad G, Watson DR, Kelso JA (2012a) Electrophysiological signatures of intentional social coordination in the 10-12 Hz range. Neuroimage 59:1795-1803.

Naeem M, Prasad G, Watson DR, Kelso JA (2012b) Functional dissociation of brain rhythms in social coordination. Clin Neurophysiol 123:1789-1797.

Noy L, Dekel E, Alon U (2011) The mirror game as a paradigm for studying the dynamics of two people improvising motion together. Proc Natl Acad Sci U S A 108:20947-20952.

Oostenveld R, Fries P, Maris E, Schoffelen JM (2011) FieldTrip: Open source software for advanced analysis of MEG, EEG, and invasive electrophysiological data. Comput Intell Neurosci 2011:156869.

Pecenka N, Keller PE (2011) The role of temporal prediction abilities in interpersonal sensorimotor synchronization. Exp Brain Res 211:505-515. 
Pfurtscheller G, Lopes da Silva FH (1999) Event-related EEG/MEG synchronization and desynchronization: basic principles. Clin Neurophysiol 110:1842-1857.

Sacheli LM, Tidoni E, Pavone EF, Aglioti SM, Candidi M (2013) Kinematics fingerprints of leader and follower role-taking during cooperative joint actions. Exp Brain Res.

Saito DN, Tanabe HC, Izuma K, Hayashi MJ, Morito Y, Komeda H, Uchiyama H, Kosaka H, Okazawa H, Fujibayashi Y, Sadato N (2010) "Stay tuned": interindividual neural synchronization during mutual gaze and joint attention. Front Integr Neurosci 4:127.

Sanger J, Muller V, Lindenberger U (2012) Intra- and interbrain synchronization and network properties when playing guitar in duets. Front Hum Neurosci 6:312.

Sanger J, Muller V, Lindenberger U (2013) Directionality in hyperbrain networks discriminates between leaders and followers in guitar duets. Front Hum Neurosci $7: 234$.

Schilbach L, Timmermans B, Reddy V, Costall A, Bente G, Schlicht T, Vogeley K (2013) Toward a second-person neuroscience. Behav Brain Sci 36:393-414.

Schippers MB, Roebroeck A, Renken R, Nanetti L, Keysers C (2010) Mapping the information flow from one brain to another during gestural communication. Proc Natl Acad Sci U S A 107:9388-9393.

Sebanz N, Bekkering H, Knoblich G (2006) Joint action: bodies and minds moving together. Trends Cogn Sci 10:70-76.

Stauffer HB (2008) An Introduction to Generalized Linear Models: Logistic Regression Models. Contemporary Bayesian and Frequentist Statistical Research Methods for Natural Resource Scientists 155-190.

Stephens GJ, Silbert LJ, Hasson U (2010) Speaker-listener neural coupling underlies successful communication. Proc Natl Acad Sci U S A 107:14425-14430.

Sui J, Rotshtein P, Humphreys GW (2013) Coupling social attention to the self forms a network for personal significance. Proc Natl Acad Sci U S A 110:7607-7612.

Tognoli E, Lagarde J, DeGuzman GC, Kelso JA (2007) The phi complex as a neuromarker of human social coordination. Proc Natl Acad Sci U S A 104:81908195.

Verhagen L, Dijkerman HC, Medendorp WP, Toni I (2012) Cortical dynamics of sensorimotor integration during grasp planning. J Neurosci 32:4508-4519.

Verhagen L, Dijkerman HC, Medendorp WP, Toni I (2013) Hierarchical organization of parietofrontal circuits during goal-directed action. J Neurosci 33:6492-6503.

Vesper C, van der Wel RP, Knoblich G, Sebanz N (2013) Are You Ready to Jump? Predictive Mechanisms in Interpersonal Coordination. J Exp Psychol Hum Percept Perform.

Wallstrom GL, Kass RE, Miller A, Cohn JF, Fox NA (2004) Automatic correction of ocular artifacts in the EEG: a comparison of regression-based and componentbased methods. Int J Psychophysiol 53:105-119.

Wing AM, Endo S, Bradbury A, Vorberg D (2014) Optimal feedback correction in string quartet synchronization. J R Soc Interface 11:20131125.

Yun K, Watanabe K, Shimojo S (2012) Interpersonal body and neural synchronization as a marker of implicit social interaction. Sci Rep 2:959. 
